\title{
Effect of Surface Morphology of ZnO Layers Deposited on Quartz Crystal Microbalance on Their Sensing Behavior during Ethanol Detection
}

\author{
Masahiro Kinoshita, ${ }^{*}$ Sergei A. Kulinich, ${ }^{2}$ Kazutoshi Noda, ${ }^{3}$ and Satoru Iwamori ${ }^{1,2}$ \\ ${ }^{1}$ Graduate School of Science and Technology, Tokai University, \\ 4-1-1 Kitakaname, Hiratsuka, Kanagawa 2591292, Japan \\ ${ }^{2}$ Research Institute of Science and Technology, Tokai University, Hiratsuka, Kanagawa 259-1292, Japan \\ ${ }^{3}$ National Institute of Advanced Industrial Science and Technology (AIST), \\ 16-1 Onogawa, Tsukuba, Ibaraki 3058569, Japan
}

(Received April 20, 2018; accepted August 20, 2018)

Keywords: $\mathrm{ZnO}$, sputtering, laser ablation in liquid, QCM, gas sensing

In this work, we compare the performance characteristics of two different zinc oxide films layered on a quartz crystal microbalance (QCM) as gas-sensing elements. The films were prepared by different methods, i.e., RF sputtering on a QCM surface and drop-casting a colloid of $\mathrm{ZnO}$ nanoparticles generated via laser ablation in liquid. Prior to use, both films were characterized in terms of their surface morphology and chemistry, after which their gas sensing properties were evaluated by using the QCM method. The film prepared from a laser-generated material was much more porous than its sputtered counterpart. Gas sensing of ethanol using the QCM coated with the two films showed that the sensing response differs depending on the state of the detection film. This demonstrated that there is a correlation between the surface characteristics and the gas adsorption characteristics of the sensors based on two different $\mathrm{ZnO}$ materials.

\section{Introduction}

In modern industry, as well as in our daily life, numerous volatile organic compounds (VOCs) are potentially harmful and can affect human health and activity. VOC gases are also one of the causes of photochemical smog around us, being released from gasoline, adhesive liquids, printing ink, cleaning agents, paints, and so on. ${ }^{(1-10)}$ Most of such gases are typically invisible, and therefore their detection by special devices, i.e., sensors (often based on semiconductor nanomaterials), becomes necessary to examine their availability and quantify their concentration in air. Semiconductor gas sensors, whose work exploits the ability of a semiconductor surface to change its resistance in the presence of a target gas, are widely used these days, mainly with metal-oxide films as detection elements. Such sensing elements have working temperatures typically between 100 and $300{ }^{\circ} \mathrm{C}$. Therefore, the development of alternative gas-sensing systems, which work at room temperature, is still in high demand. ${ }^{(3,11,12)}$

*Corresponding author: e-mail: 6btad001@mail.u-tokai.ac.jp

https://doi.org/10.18494/SAM.2018.1964 
There is another approach that exploits a quartz crystal microbalance (QCM) operating at room temperature and relying on the mass change of the QCM membrane that absorbs gas molecules. ${ }^{(13-25)}$ Among the top layers coated onto the QCM, zinc oxide materials are also used as detection films. ${ }^{(14-25)}$ It is expected that, depending on their preparation methods, their sensing properties will be affected by their roughness and surface morphology. The reason for using zinc oxide as the top layer on the QCM is that it is easy to prepare and control its surface morphology, as well as to compare its performance with those of other sensors based on $\mathrm{ZnO}$ and previously reported in the literature. ${ }^{(14-25)}$

In this work, the surface properties of two different $\mathrm{ZnO}$ films prepared by different techniques were evaluated by using the QCM. More specifically, the ZnO layers studied here were deposited directly on the QCM surface either by radiofrequency (RF) sputtering or by drop-casting from a $\mathrm{ZnO}$ colloid prepared by laser ablation in liquid (LAL). ${ }^{(3,28-33)}$ The two $\mathrm{ZnO}$ nanomaterials gave rise to films with a relatively smooth or porous surface morphology, respectively. This allowed us to test two samples with contrasting gas adsorption characteristics. The absorption and desorption behaviors of the target gas on such $\mathrm{ZnO}$ surfaces were expected to be different, as some gases could remain much longer inside the rougher surface. It was indeed confirmed that the more porous film demonstrated a somewhat different detection behavior, which is related to its larger surface area and porosity.

\section{Experimental Section}

\subsection{QCM method}

When an $\mathrm{AC}$ electric field is applied to the quartz oscillator, the oscillator vibrates at a certain frequency. ${ }^{(13-27)}$ When a substance is adsorbed on the detection membrane, a change in frequency occurs depending on the mass of the substance. The amount of adsorbed substance is expressed using the Sauerbrey equation. ${ }^{(1)}$ As the amount of adsorbed substance increases, the frequency decreases, and when the amount of adsorbed substance decreases, the frequency increases. ${ }^{(13-27)}$ Hence, the change in frequency can be detected, after which the change in the amount of gas adsorbed on the quartz oscillator is measured. ${ }^{(13)}$ Equation (1) below shows how the difference in frequency caused by absorption $(\Delta F)$ relates to the mass of the absorbed gas $(\Delta m)$, the fundamental frequency of the QCM $\left(F_{0}\right)$, the surface area $(A)$, the shear stress of the quartz crystal $(\mu)$, and its density $(\rho)$.

$$
\Delta F=\frac{2 \times F_{0}{ }^{2}}{A \times \sqrt{\mu \times \rho}} \times \Delta m
$$

\subsection{LAL}

As a technique, LAL is an easy-to-use, environmentally friendly, and convenient physical method of preparing various nanomaterials at the laboratory scale..$^{(3,11,28-33)}$ It exploits the laser beam focused on a solid target immersed in a liquid medium. Upon hitting the target 
surface, the high-energy laser pulse generates plasma (or liquid-metal droplets), which then reacts with the liquid medium, becomes quenched, and condenses as various nanostructures (whose morphology, size, and chemical composition depend on laser parameters, target, and liquid). ${ }^{(1,12,28-33)}$ Among other materials, this method is known to be efficient for the preparation of various metal oxide nanostructures, including $\mathrm{ZnO} .^{(3,28-33)}$ This explains why it was chosen in this study as one of the preparation approaches that provide $\mathrm{ZnO}$ colloids, which were drop-cast as films on the QCM. The setup used in this study is presented in Fig. 1, while more details on procedures can be found elsewhere. ${ }^{(3,29-31)}$ The laser used was a nanosecond pulsed laser with a pulse duration of $7 \mathrm{~ns}$, a frequency of $10 \mathrm{~Hz}$, an ablation time of $30 \mathrm{~min}$, a pulse energy of $50 \mathrm{~mJ} /$ pulse, and a wavelength of $1064 \mathrm{~nm}$. A quartz cuvette was filled with pure water and a zinc plate was fixed on its wall (Fig. 1). A laser beam was projected horizontally onto the surface of the zinc plate, the focal point being adjusted using a lens. A stirring bar was placed at the bottom of the cuvette, and water was agitated during the ablation process. The prepared $\mathrm{ZnO}$ nanoparticles were collected after separation using a centrifuge.

\subsection{RF sputtering of $\mathrm{ZnO}$ film}

For the sputtering method, a zinc plate was placed on the target and a crystal oscillator (QCM) was placed on the opposite side. Oxygen was introduced into the chamber after the evacuation of air in the chamber, and the operating pressure was adjusted. During sputtering, pure oxygen $(99.5 \%)$ was used as atmosphere inside the chamber. The main parameters used during sputter deposition are given in Table 1.

\subsection{Drop-casting $\mathrm{ZnO}$ colloid}

A few microliters of fresh colloidal solution with $\mathrm{ZnO}$ nanoparticles prepared by LAL was deposited using a pipette on the QCM surface and then dried on a hotplate at $80{ }^{\circ} \mathrm{C}$ for 30 min. Upon drop-casting, the sample was annealed in air for $2 \mathrm{~h}$ at $200{ }^{\circ} \mathrm{C}$. This temperature was previously found to be the maximum at which QCMs survive without any deterioration in

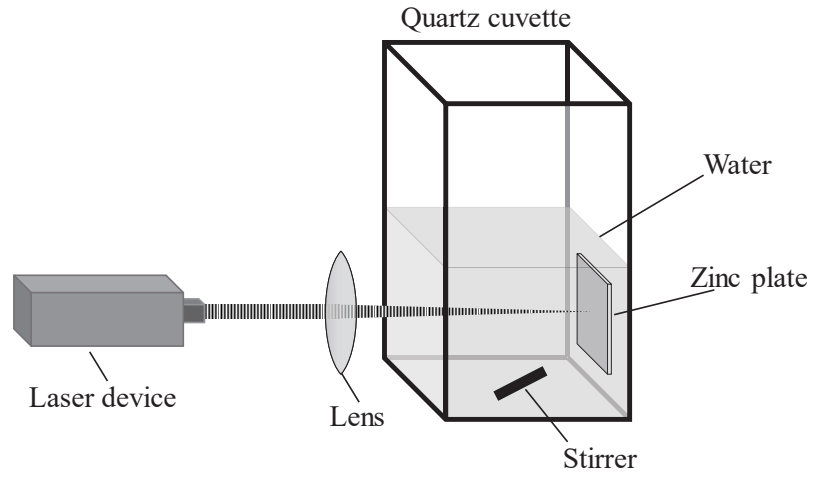

Fig. 1. Schematic presentation of LAL.
Table 1

Experimental conditions during RF sputter deposition.

\begin{tabular}{cc}
\hline $\begin{array}{c}\text { Operating pressure } \\
(\mathrm{Pa})\end{array}$ & $\begin{array}{c}\text { Oxygen inflow amount } \\
(\mathrm{ml} / \mathrm{min})\end{array}$ \\
\hline 4 & 20 \\
\hline
\end{tabular}


their properties. The annealing was performed to stabilize the $\mathrm{ZnO}$ layer drop-cast onto the gold electrode surface and improve its adhesion as a detection film. The aggregation of $\mathrm{ZnO}$ nanoparticles is known to occur at higher temperatures, resulting in larger grain growth, ${ }^{(6)}$ that is why annealing was performed at such a low temperature.

\subsection{Sample gas preparation}

Figure 2 shows the setup used to prepare a target gas, the system being based on vaporization. A cotton tissue (Bemcot) previously immersed in ethanol was placed in a bin where it started releasing ethanol gas into the volume. After that, water surrounding the bin was set to $30{ }^{\circ} \mathrm{C}$. The base gas was introduced at a rate of $100 \mathrm{ml} / \mathrm{min}$ for a fixed period of time, and ethanol was vaporized and collected in a gas sampling bag. The gas concentration in the sampling bag was measured using standard gas testing tubes and then diluted to a specified concentration. Gas sensing characteristics due to the gas concentration difference were previously reported to be linear in the range of 250 to $2500 \mathrm{ppm}^{(7)}$ In this study, the gas sensing characteristics of the two above-mentioned samples were tested by changing the gas concentration in the test chamber and monitoring the frequency change $(\Delta F)$ of the samples. Ethanol was chosen as a model target gas to clarify the relationship between the gas adsorption and surface topography of the two $\mathrm{ZnO}$ films with contrasting surface roughnesses. In addition, ethanol is known to be easy in handling and use, and it was previously detected by $\mathrm{ZnO}$-coated QCM sensors. ${ }^{(9,10)}$

\subsection{Gas sensing experiments}

Gas sensing tests were carried out in the setup schematically shown in Fig. 3. The temperature was set to $27{ }^{\circ} \mathrm{C}$ to minimize the influence of outside air. The QCM membranes used had a frequency of around $9 \mathrm{MHz}$, with an electrode of $5 \mathrm{~mm}$ diameter. A gas sampling bag containing ethanol gas was connected to a valve tube. To take a reference value of the gas, a bottle containing silica gel was connected to the sampling bag that contains the base gas. The crystal resonators were attached to ceramic cells at intervals of about $8 \mathrm{~mm}$ and connected to computers through probes. The flowing gases were removed at a rate of $100 \mathrm{ml} / \mathrm{min}$ each. The frequency change was recorded using the software QCMeasur. To measure the frequency

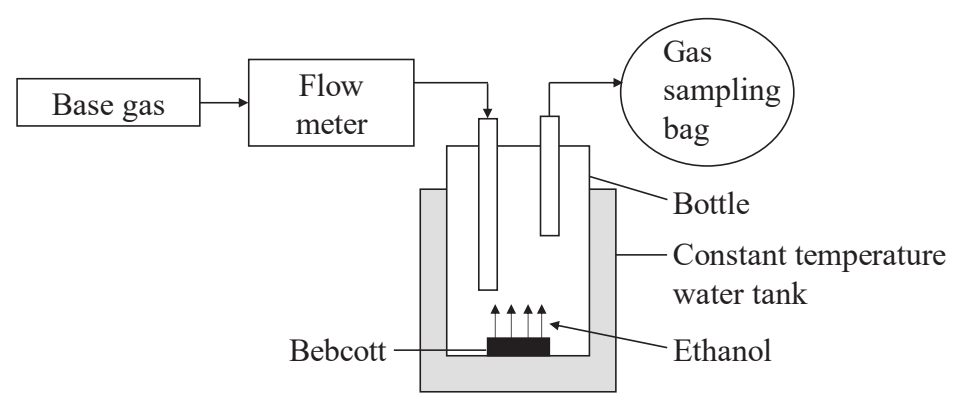

Fig. 2. Experimental setup used to prepare and collect gas samples. 


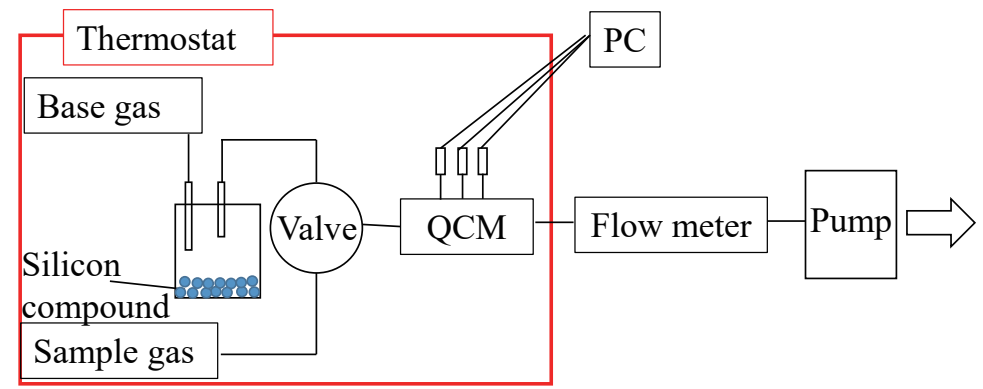

Fig. 3. (Color online) Experimental setup for estimation of adsorption mass of VOC gases.

change in the presence of the sample gas, we measured the stable frequency rate under the base gas and then set it as a start value. After measuring for $60 \mathrm{~s}$ with the base gas, the valve was closed and opened repeatedly and the sample gas was flown in for $600 \mathrm{~s}$ to be adsorbed. After measuring for $600 \mathrm{~s}$, the base gas was introduced again, and the trend was double-checked and confirmed.

\section{Results and Discussion}

\subsection{Atomic force microscopy (AFM) results}

The obtained AFM results are presented in Fig. 4, where panels (a) and (b) show the surface images of RF-sputtered and drop-cast samples, respectively. The average roughness $R_{a}$ values of sputtered and LAL-prepared samples [see Figs. 4(a) and 4(b)] were 0.86 and $38.81 \mathrm{~nm}$, respectively. From Figs. 4(a) and 4(b), it is clearly seen that the two films had very different surface morphologies. As seen in Fig. 4(a), the RF-sputtered (SP) sample had a dense surface morphology and was relatively smooth. In contrast, the sample in Fig. 4(b) had a much rougher surface, with more surface pores and hills. The latter sample is thus concluded to have a larger surface area than its sputtered counterpart.

\subsection{Scanning electron microscopy (SEM) results}

Figure 5 shows the surface images of the same samples taken at different magnifications: panels $(\mathrm{a}-\mathrm{c})$ exhibit the surface of the sample drop-cast from the LAL-generated $\mathrm{ZnO}$ colloid, while panels $(\mathrm{d}-\mathrm{f})$ demonstrate the surface of the RF-sputtered sample. In agreement with Fig. 4, it is well seen that the sputtered sample was much smoother than the drop-cast one. Accordingly, many more physical adsorption sites are expected on the latter sample, which is much rougher and much more porous.

\subsection{X-ray photoelectron spectroscopy (XPS) results}

The results of XPS measurements are presented in Figs. 6(a) and 6(b), where narrow-scan (a) Zn2p3/2 and (b) O1s spectra are given for both samples. From Fig. 6(a), both samples were 


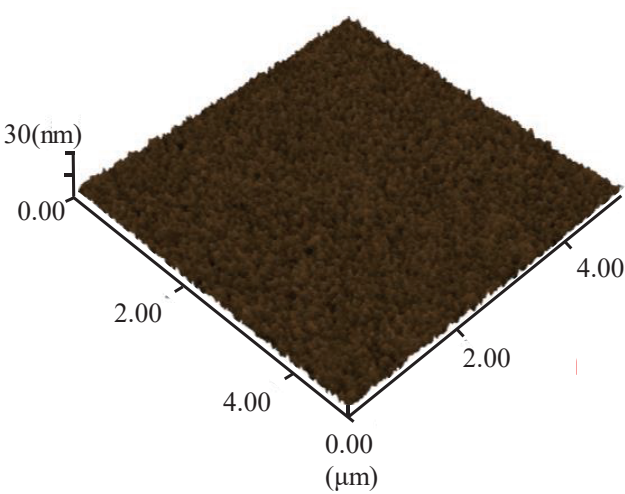

(a)

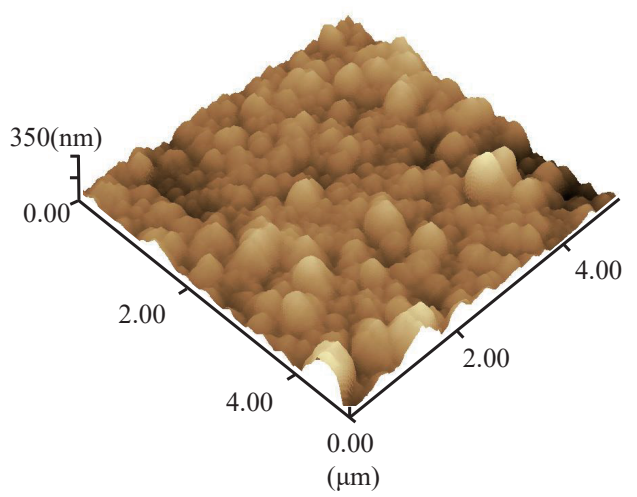

(b)

Fig. 4. (Color online) AFM surface images of samples prepared by (a) sputtering and (b) drop-casting of LALproduced $\mathrm{ZnO}$ colloid.

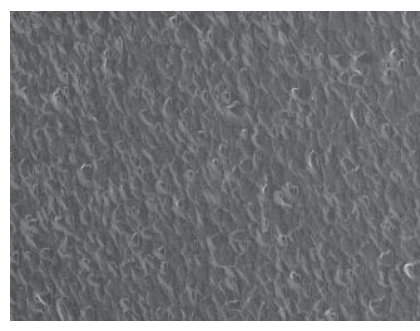

(a)

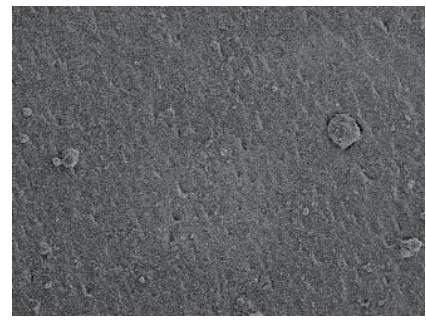

(d)

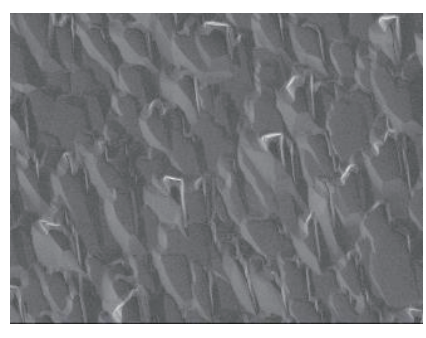

(b)

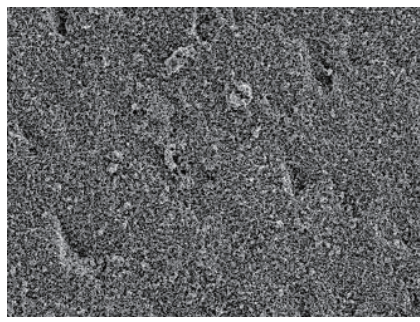

(e)

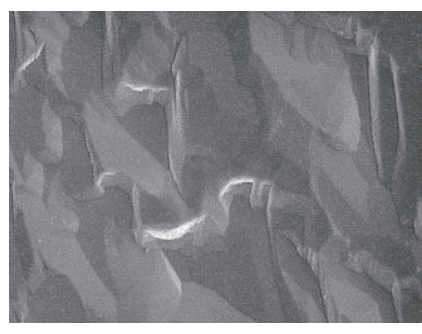

(c)

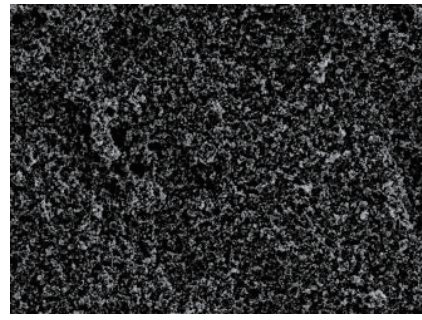

(f)

Fig. 5. SEM surface images (taken at different magnifications) of samples prepared by (a-c) magnetron sputtering and (d-f) drop-casting of LAL-produced $\mathrm{ZnO}$ colloid.

concluded to consist of $\mathrm{ZnO}$ because their peaks are seen to be symmetrical and have their top near $1021 \mathrm{eV}^{(34)}$ The component observed at $532 \mathrm{eV}$ in Fig. 6(b) was assigned to surfaceadsorbed water or hydroxyl groups. ${ }^{(35)}$ Since the LAL-generated $\mathrm{ZnO}$ nanoparticles were prepared in water, their higher degree of surface hydroxylation (shoulder at $532 \mathrm{eV}$ ) compared with the sputtered sample was well expected. It is thus concluded from Fig. 6 that both samples were composed of $\mathrm{ZnO}$ nanoparticles, the latter having some degree of surface hydroxilation. ${ }^{(12,29)}$ The formation of such $\mathrm{ZnO}$ nanoparticles from metal $\mathrm{Zn}$ during both $\mathrm{RF}$ sputtering in oxide or LAL in water was well expected since $\mathrm{Zn}$ has a low melting point and is known to be easily oxidized. 


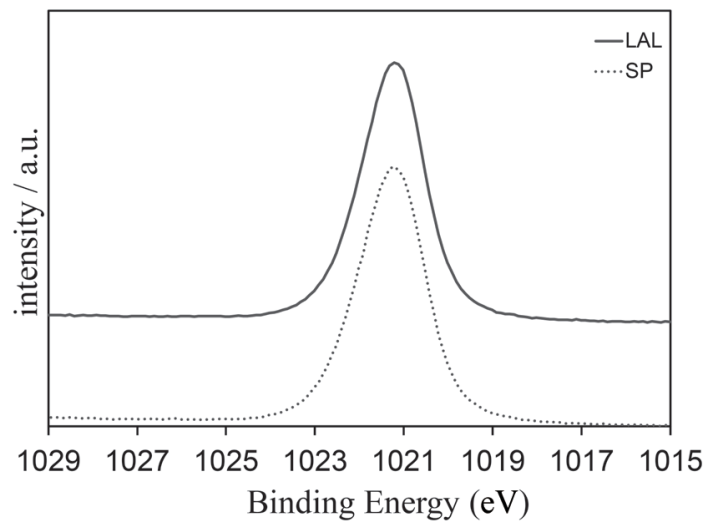

(a)

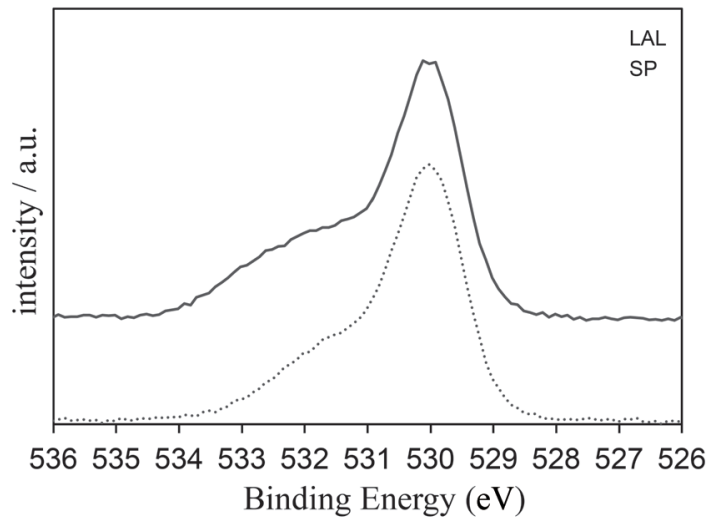

(b)

Fig. 6. XPS (a) Zn2p3/2 and (b) O1s spectra of samples SP-prepared (lower curve) and drop-cast from LALgenerated nanoparticles (upper curve).

\subsection{Gas sensing results}

Figure 7 shows how the frequency of the two QCM samples changed over time when ethanol (with a concentration of $500 \mathrm{ppm}$ ) was added into the chamber. It this work, both samples deposited on QCM membranes were prepared with comparable masses (5.94 and $4.94 \mu \mathrm{g}$, respectively, for the LAL-generated $\mathrm{ZnO}$ nanomaterial and sputtered $\mathrm{ZnO}$ ) as we intended to reduce the possible effect of $\mathrm{ZnO}$ mass and focus on the surface morphology of the two samples. The results shown in Fig. 7 are presented as $\Delta F$ per coating mass, which was done to minimize the mass effect even more. It is seen that both QCM devices (coated with both types of $\mathrm{ZnO}$ material) demonstrated similar behaviors. Although the film prepared by the SP method (lower curve in Fig. 7) is seen to have lower detection characteristics, it also demonstrates a smaller detection error than its counterpart prepared based on a laser-generated $\mathrm{ZnO}$ nanomaterial. Based on the curves presented in Fig. 7, we can conclude that the concentration of ethanol used in the two experiments (at $500 \mathrm{ppm}$ ) was higher than the actual sensitivity limit of the two sensors. Because of the preparation technique adopted in this study (see Sect. 2.5 and Fig. 2 above), it was difficult to prepare ethanol samples with lower concentrations; thus, further optimization and improvement of the devices are underway. Moreover, $\mathrm{ZnO}$ sensors reported before (both chemiresistive and based on QCM) demonstrated sensitivity to ethanol as low as $15-50 \mathrm{ppm},{ }^{(3,22)}$ which is still quite challenging for the devices discussed in this work.

Figure 8 shows how two samples based on LAL-prepared $\mathrm{ZnO}$ with different membrane masses $(5.94$ and $7.06 \mu \mathrm{g})$ responded to the same ethanol concentration. The behavior of the two curves in Fig. 8 is seen to be comparable, and the lighter sample appears to be even slightly more sensitive than its heavier counterpart. This implies that the experimental approach used in this study indeed allowed us to minimize the effect of $\mathrm{ZnO}$ mass, and the difference between the two curves observed in Fig. 7 is explained by different surface morphologies of the two samples. After measuring both ethanol concentrations tested (500 and $3000 \mathrm{ppm}$ ), the sputterprepared $\mathrm{ZnO}$ film regained its initial frequency in $10 \mathrm{~min}$ upon switching to the base gas 


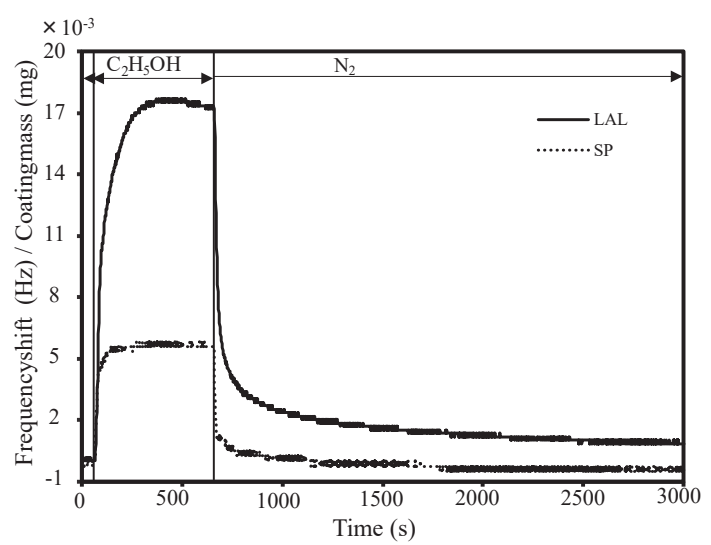

Fig. 7. Ethanol gas sensing performance of the sputter- (lower curve) and drop-cast prepared (upper curve) samples (ethanol concentration: $500 \mathrm{ppm}$ ). The membrane mass of the sensors was 5.94 (LAL) and 4.94 $\mu \mathrm{g}(\mathrm{SP})$.

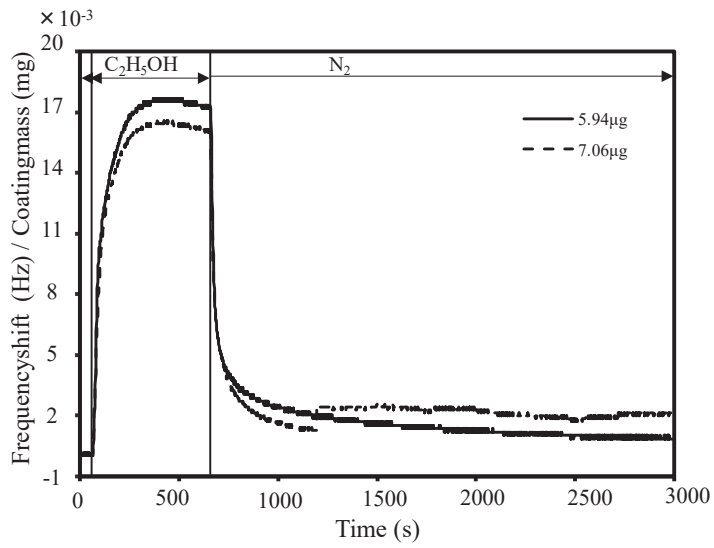

Fig. 8. Ethanol gas sensing performance of dropcast prepared samples with different membrane mass of 5.94 and $7.06 \mu \mathrm{g}$ (ethanol concentration: $500 \mathrm{ppm}$ ).

atmosphere (see curves in Fig. 7 for 500 ppm). At the same time, under the same conditions, the QCM coated with the film formed by the LAL-generated nanoparticles did not reach the initial value of the base gas, the difference being larger at a higher detection concentration of the target gas (16 and 10\% differences after measuring 3000 and $500 \mathrm{ppm}$, respectively). This finding can also be explained by the larger surface area of the more porous sample prepared via dropcasting. It is reasonable to assume that ethanol molecules absorbed onto its pore surface will be washed out with some delay when the atmosphere in the chamber is replaced by an ethanol-free one.

Our results thus allow us to conclude that the higher sensitivity demonstrated by the $\mathrm{ZnO}$ film drop-cast from laser-generated nanoparticles is due to its higher porosity and larger surface area, which were well documented by both AFM and SEM analyses [see Figs. 4(a), 4(b), and 5(a)-5(c)] It is also worth mentioning that the LAL-produced nanomaterials are well known to have more surface defects, which result in large temperature differences during quenching (and particle formation) and are favorable as surface absorption sites for gas molecules. ${ }^{(27-31)}$ At the same time, this also explains the lower desorption rates on such surfaces.

\section{Conclusions}

In this work, we studied the gas sensing characteristics and surface morphology of $\mathrm{ZnO}$ films deposited on a QCM by RF sputtering or drop-casting of nanoparticles prepared by laser ablation in water. Both samples demonstrated similar chemical states of elements, as both films deposited on the QCM were based on $\mathrm{ZnO}$. However, the surface morphologies of the two membranes were different. Correspondingly, the gas sensing characteristics of the samples were somewhat different. The sample prepared from laser-produced $\mathrm{ZnO}$ nanoparticles was more sensitive than its sputtered counterpart. This can be explained by the much higher 
porosity of the former $\mathrm{ZnO}$ film than the latter, which is believed to result in more adsorption sites and thus a higher sensitivity. Thus, the correlation between the surface characteristics, such as roughness and porosity, and the gas adsorption characteristics of the same $\mathrm{ZnO}$ surface was demonstrated using a QCM.

\section{References}

1 I.-D. Kim, A. Rothshild, and H. L. Tuller: Acta Mater. 61 (2013) 974.

2 Y.-B. Hahn, R. Ahmad, and N. Tripathy: Chem. Commun. 48 (2012) 10369.

3 T. Kondo, Y. Sato, M. Kinoshita, P. Shankar, N. N. Mintcheva, M. Honda, S. Iwamori, and S. A. Kulinich: Jpn. J. Appl. Phys. 56 (2017) 080304.

4 J. Xie, H. Wang, Y. Lin, Y. Zhou, and Y. Wu: Sens. Actuators, B 177 (2013) 1083.

5 Y. Q. Zhang, Z. Li, T. Ling, S. A. Kulinich, and X. W. Du: J. Mater. Chem. A 4 (2016) 8700.

6 J. Eriksson, V. Khranovskyy, F. Söderlind, P. O. Käll, R. Yakimova, and A. L. Spetz: Sens. Actuators, B 137 (2009) 94.

7 L. Bie, X. Yan, J. Yin, Y. Duan, and Z. Yuan: Sens. Actuators, B 126 (2007) 604.

8 X. H. Liu, P. F. Yin, S. A. Kulinich, Y. Z. Zhou, J. Mao, T. Ling, and X. W. Du: ACS Appl. Mater. Interfaces 9 (2017) 602.

9 H. Xu, X. Liu, D. Cui, M. Li, and M. Jiang: Sens. Actuators, B 114 (2006) 301.

10 F. Paraguay, M. Miki-Yoshida, J. Morales, J. Solis, M. Yoshida, U, J. Morales, J. Solis, and W. Estrada: Thin Solid Films 373 (2000) 137.

11 M. Honda, T. Kondo, T. Owashi, P. Shankar, S. Iwamori, Y. Ichikawa, and S. A. Kulinich: New J. Chem. 41 (2017) 11308.

12 N. Mintcheva, A. A. Aljulaih, S. Bito, M. Honda, T. Kondo, S. Iwamori, and S. A. Kulinich: J. Alloy. Compd. 747 (2018) 166.

13 S. Iwamori, K. Yoshino, H. Matsumoto, K. Noda, and I. Ishiyama: Sens. Actuators, B 171-172 (2012) 769.

14 C. C. Wang, P. Y. Lin, C. J. Lu, and M. H. Liu: Instrum. Sci. Technol. 45 (2017) 639.

15 N. Horzum, D. Tascioglu, C. Ozbek, S. Okur, and M. M. Demir: New J. Chem. 38 (2014) 5761.

16 T. Ito, Y. Fujii, N. Yamanishi, N. Asai, T. Shimizu, and S. Shingubara: Procedia Eng. 168 (2016) 411.

17 X. H. Wang and J. Zhang: Appl. Mech. Mater. 248 (2013) 199.

18 K. Lovchinov, M. Petrov, M. Ganchev, V. Georgieva, H. Nichev, B. Georgieva, and D. Dimova-Malinovska: J. Phys. Conf. Ser. 514 (2014) 012023.

19 Z. Yuan, H. Tai, X. H. Bao, C. C. Liu, Z. B. Ye, and Y. D. Jiang: Mater. Lett. 174 (2016) 28.

20 N. Sakly, A. Haj Said, and H. Ben Ouada: Mater. Sci. Semicond. Process. 27 (2014) 130.

21 S. Oztur, A. Kosemen, Z. A. Kosemen, N. Kilinc, Z. Z. Ozturk, and M. Penza: Sens. Actuators, B 222 (2016) 280.

22 J. Xie, H. Wang, and M. Duan: Sens. Actuators, B 203 (2014) 239.

23 J. Xie, H. Wang, Y. H. Lin, Y. Zhou, and Y. P. Wu: Sens. Actuators, B 177 (2013) 1083.

24 S. I. Boyadjiev, V. Georgieva, R. Yordanov, Z. Raicheva, and I. M. Szilagyi: Appl. Surf. Sci. 387 (2016) 1230.

25 V. A. Minh, L. A. Tuan, T. Q. Huy, V. N. Hung, and N. V. Quy: Appl. Surf. Sci. 265 (2013) 458.

26 W. A. Lazier and H. Adkins: J. Phys. Chem. 30 (1926) 353.

27 Y. Fjita and T. Kwan: Bull. Chem. Soc. Jpn. 31 (1958) 850.

28 H. B. Zeng, X. W. Du, S. C. Singh, S. A. Kulinich, S. K. Yang, J. P. He, and W. P. Cai: Adv. Funct. Mater. 22 (2012) 1333.

29 M. Honda, T. Goto, T. Owashi, A.G. Rozhin, S. Yamaguchi, T. Ito, and S.A. Kulinich: Phys. Chem. Chem. Phys. 18 (2016) 23628.

30 S. A. Kulinich, T. Kondo, Y. Shimizu, and T. Ito: J. Appl. Phys. 113 (2013) 033509.

31 T. Goto, M. Honda, S. A. Kulinich, Y. Shimizu, and T. Ito: Jpn. J. Appl. Phys. 54 (2015) 070305.

32 K. Y. Niu, J. Yang, S. A. Kulinich, J. Sun, H. Li, and X. W. Du: J. Am. Chem. Soc. 132 (2010) 9814.

33 N. Mintcheva, A. A. Aljulaih, W. Wunderlich, S. A. Kulinich, and S. Iwamori: Materials 11 (2018) 1127.

34 R. A. Gaashani, S. Rasiman, A. R. Daud, N. Tabet, and Y. A. Durin: Ceram. Int. 39 (2013) 2283.

35 M. Chena, X. Wang, Y. H. Yu, Z. L. Pei, X. D. Bai, C. Sun, R. F. Huang, and L. S. Wen: Appl. Surf. Sci. 158 (2000) 134. 